\title{
Membrane effects of aminoglycoside antibiotics measured in liposomes containing the fluorescent probe, 1-anilino-8-naphthalene sulfonate
}

\author{
S. Au ${ }^{a}$, J. Schacht ${ }^{b}$ and N. Weiner ${ }^{a}$ \\ "College of Pharmacy and ${ }^{h}$ Kresge Hearing Research Institute, University of Michigan, Ann Arbor, MI 48109 (U.S.A.)
}

(Received 14 May 1986)

\begin{abstract}
Key words: Aminoglycoside; Antibiotics - membrane interaction; Liposome; Phosphatidylinositol bisphosphate;
\end{abstract} Gentamicin; Fluorescent probe

The mechanism of membrane disturbance by aminoglycoside antibiotics was investigated in liposomes containing the fluorescent probe, 1-anilino-8-naphthalene sulfonate (ANS). Liposomes of PC and different anionic phospholipids (1:1 to 15:1 molar ratios) were challenged with aminoglycosides in the presence of low $(1 \mu \mathrm{M})$ and high $(3 \mathrm{mM})$ concentrations of calcium. Liposomes containing $\mathrm{PIP}_{2}$ showed the greatest drug-induced changes in ANS fluorescence in the presence of high and low concentrations of calcium and at all PC: PIP $P_{2}$ molar ratios tested. Liposomes containing other anionic phospholipids (PS, PI and PIP) were not reactive toward aminoglycosides in the presence of $3 \mathrm{mM}$ calcium or when the ratio of $P C$ to anionic lipid was increased to $10: 1$. The aminoglycoside-induced changes of ANS fluorescence were not due to any changes in the emission spectrum of ANS, nor to changes in quantum yield, nor to a change in the binding affinity of ANS. It is concluded that a specific aminoglycoside-PIP $\mathbf{P}_{2}$ interaction results in phase separation of $\mathrm{PC}$ and $\mathrm{PIP}_{2}$ and thus increases the number of available ANS binding sites in PC: PIP ${ }_{2}$ liposomes.

\section{Introduction}

Despite recent progress in the management of bacterial infections, most prominently the introduction of the powerful cephalosporins, the aminoglycoside antibiotics are still indispensable antimicrobial agents. The search for replacement drugs is prompted by increasing bacterial resistance and the adverse effects of the aminoglycosides on the function of the inner ear (both hearing and equilibrium) and the kidney. The efficient development of improved aminoglycosides and semi-synthetic derivatives is hampered by the lengthy and complex animal experiments necessary to evaluate drug-induced hearing loss. Ra-

Correspondence: Dr. N. Weiner, College of Pharmacy, University of Michigan, Ann Arbor, MI 48109, U.S.A. tional design of new drugs would be greatly aided by model systems in which in-vitro correlates of toxicity could be tested. Recent advances in our understanding of aminoglycoside toxicity have enabled us to propose such model systems. They are derived from a key reaction in the proposed multi-step toxic mechanism, the specific binding of aminoglycosides to phosphatidylinositol bisphosphate $\left(\mathrm{PIP}_{2}\right)$ [1].

In vivo experiments as well as studies with monolayers and liposomes as model membranes have demonstrated the unique nature of the interactions between aminoglycosides and $\mathrm{PIP}_{2}$. When monomolecular films of $\mathrm{L}$ - $\alpha$-phosphatidylcholine mixed with various anionic phospholipids were challenged with aminoglycosides, only the $\mathrm{PIP}_{2}$ containing films showed a dose-dependent and toxicity-related increase in surface pressure while others showed a decrease or no significant effect 
[2]. Aminoglycosides also lowered the phase transition temperature of $\mathrm{PIP}_{2}$ containing liposomes while it raised the transition temperature of all other anionic phospholipid-PC liposomes tested [3]. Since the membrane effects of a variety of aminoglycosides correlated well with their ototoxicity, these model systems showed promise both in elucidating molecular mechanisms and screening drugs for their toxic potential. A critical issue, however, in relating the in vitro to the in vivo situation was the fact that these models required concentrations of $\mathrm{PIP}_{2}$ that were at least one order of magnitude higher than expected in the biological target membranes. Furthermore, these models were only able to document drug-induced alterations in membrane structure and fluidity without allowing insight into the underlying mechanisms at the molecular level. The present study addresses these two points.

The fluorescent membrane probe, 1-anilino-8naphthalene sulfonate (ANS), has been widely used to study structural changes induced by a large variety of compounds in natural and artificial membranes [4]. ANS is noncovalently incorporated into these structures and its fluorescence is highly sensitive to changes in the molecular environment of the phospholipid bilayer. Its negligible interference with membrane properties and its convenient absorption characteristics make ANS the fluorescent probe of choice to study conformational changes that occur within the bilayers of liposomes as a result of interactions with drug molecules. The resultant fluorescence changes can be interpreted at the molecular level since differences in the molecular environment of the probe can be easily separated from changes in the binding affinity or number of binding sites.

\section{Materials and Methods}

$\mathbf{L}-\boldsymbol{\alpha}$-Phosphatidylcholine (PC, from frozen egg yolk), L- $\alpha$-phosphatidylserine (PS), L- $\alpha$-phosphatidylinositol (PI) and 1-anilino-8-naphthalene sulfonate (magnesium salt) were purchased from Sigma Chemical Co., St. Louis, M.O. Gentamicin sulfate was supplied by the Schering Corp. (Bloomfield, N.J.) and amikacin by Bristol Laboratories (New York, NY). Phosphatidylinositol 4- phosphate (PIP) and phosphatidylinositol 4,5-bisphosphate $\left(\mathrm{PIP}_{2}\right)$ were isolated from Sigma brain extracts (Cat. No. P6023) by chromatography on immobilized neomycin [5]. Lipids were stored in the dark under nitrogen at $-20^{\circ} \mathrm{C}$; PIP and PIP were dried and the other lipids were kept dissolved in chloroform. The purity of the lipids was grater than $98 \%$ as estimated by thin-layer chromatography. The water was thrice distilled, the second and third time in an all-glass apparatus. The buffer for all experiments was $0.05 \mathrm{M}$ Hepes ( $N$-2-hydroxyethyl-1-piperazine- $N^{\prime}$-2-ethanesulfonic acid), $\mathrm{pH} 7.0$; ionic strength 0.2 , adjusted with sodium chloride. All other chemicals were of reagent grade.

Preparation of liposomes. Multilamellar liposomes were prepared as previously described [3] from PC and an anionic phospholipid at molar ratios ranging from $1: 1$ to $15: 1$. Aliquots of the chloroform solutions of the lipid mixtures were transferred to a $10-\mathrm{ml}$ round-bottom flask. The solvent was evaporated under nitrogen while the flask was rotated by hand to aid the formation of a uniformly thin lipid film at its bottom. Residual solvent was removed by storing the flask overnight under vacuum. The dried lipid was then suspended in buffer and vortexed intermittently for 1 $h$. The final dispersion was sonicated for two minutes in a bath type sonicator (Branson Cleaning Equipment, Shelton, CT).

Fluorescence experiments. Aliquots of the liposome stock suspension were incubated with various concentrations of ANS and calcium chloride or aminoglycosides or both for $60 \mathrm{~min}$ at room temperature. Incubation for more than $60 \mathrm{~min}$ did not change the results. Fluorescence was measured at room temperature with a Perkin-Elmer, Model LS-5 spectrofluorometer. The slit width was $10 \mathrm{~nm}$ for both excitation $(385 \mathrm{~nm})$ and emission (475 $\mathbf{n m}$ ). Each experiment was conducted in triplicate and the reproducibility was always within $3 \%$. The observed fluorescence intensities of the mixtures were corrected for the individual fluorescence of the liposomes, free ANS, calcium and aminoglycosides.

The molar ratio of $\mathrm{PC}$ to anionic lipids in the liposomes was varied from $1: 1$ to $15:$. Quantum yield, dissociation constants and number of binding sites were determined at a molar ratio of $4: 1$ 
since this ratio proved most sensitive for quantitative studies [6].

\section{Results}

The absolute magnitude of the fluorescence of the ANS-treated liposomes varied with the type as well as with the quantity of the anionic phospholipid. These differences are due primarily to differences in electrostatic potentials of the liposomes [4]. In order to compare the effects of aminoglycosides or calcium on liposomes of different lipid compositions, the fluorescence intensities in the presence of drug $\left(F_{i}\right)$ were normalized to the corresponding measurement in its absence
$\left(F_{0}\right)$. It should be noted that neither calcium nor aminoglycosides, even at concentrations as high as $10 \mathrm{mM}$, affected the fluorescence of ANS in liposomes of pure PC.

Calcium at concentrations below $0.1 \mathrm{mM}$ had very little effect on the fluorescence of ANS in liposomes prepared from $\mathrm{PC}$ and any of the anionic phospholipids (1:1 molar ratio) (Fig. 1A). Higher concentrations of calcium increased fluorescence of the $\mathrm{PC} / \mathrm{PIP}_{2}$ liposomes about 2 -fold and this level was reached at $1 \mathrm{mM} \mathrm{Ca}^{2+}$. In contrast, liposomes containing PS, PI or PIP were not significantly affected by calcium even at concentrations as high as $10 \mathrm{mM}$.

The effects of gentamicin on the fluorescence
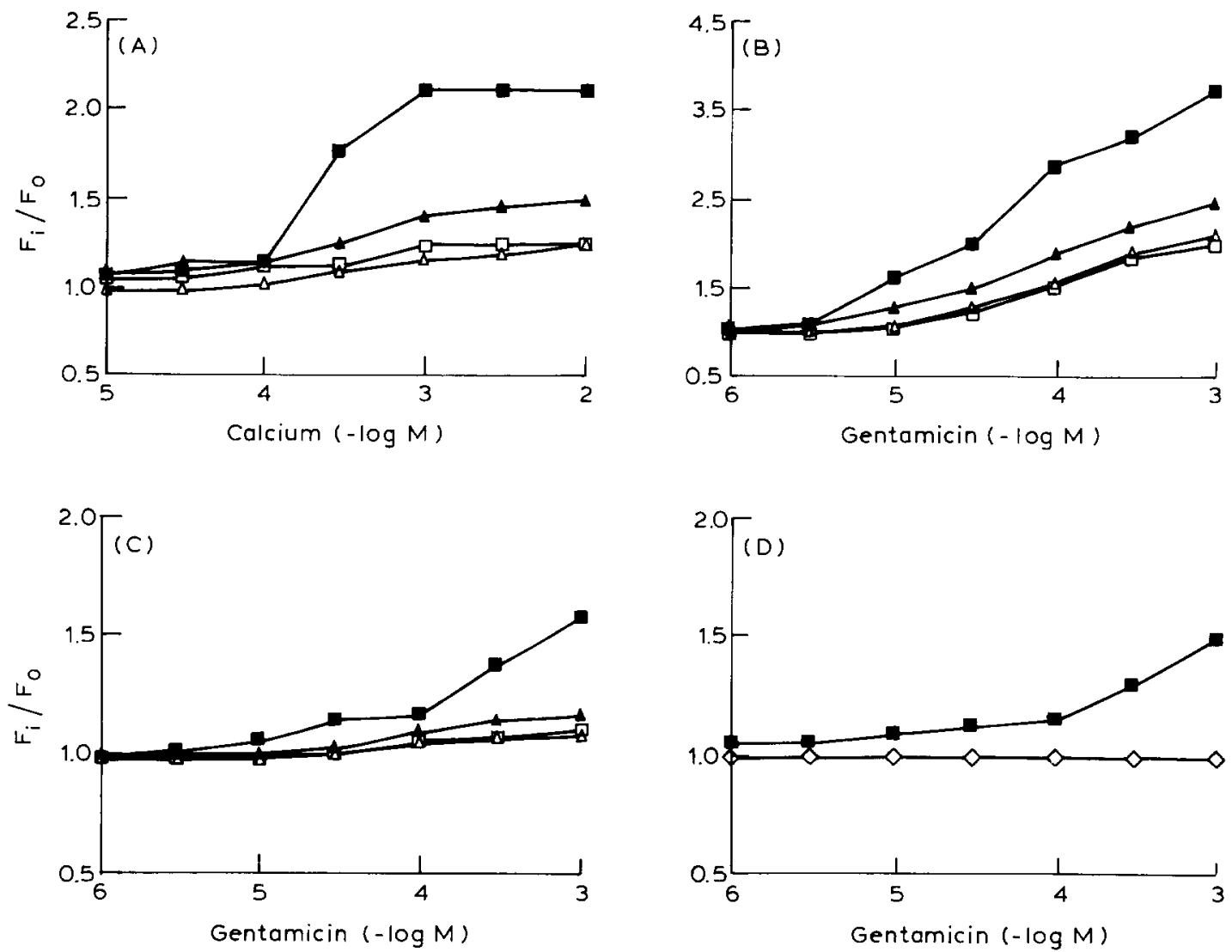

Fig. 1. Relative fluorescence intensity of mixed liposomes of PC and anionic phospholipids incubated with $10 \mu \mathrm{M}$ ANS and calcium chloride or gentamicin for $60 \mathrm{~min}$ at room temperature as described in Materials and Methods. (A) PC/anionic phospholipid (molar ratio, $1: 1$ ) liposomes incubated with calcium chloride. (B) PC/anionic phospholipid (molar ratio, $1: 1$ ) liposomes incubated with gentamicin in the presence of $1 \mu \mathrm{M}$ calcium. (C) PC/anionic phospholipid (molar ratio, 1:1) liposomes incubated with gentamicin in the presence of $3 \mathrm{mM}$ calcium. (D) PC/anionic phospholipid (molar ratio, 10:1) liposomes incubated with gentamicin in the

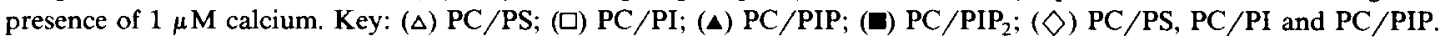


intensity of ANS-treated liposomes were first measured in the presence of $1 \mu \mathrm{M}$ calcium which is a known competitor of aminoglycoside binding (Fig. 1B). This concentration of calcium approximates intracellular levels and was considered appropriate since $\mathrm{PIP}_{2}$ is believed to reside in the inner bilayers of cytoplasmic membranes [1]. Liposomes of all lipids tested showed varying degrees of reactivity towards the aminoglycoside and the $\mathrm{PIP}_{2}$-containing liposomes displayed the greatest increase in fluorescence. In the presence of excess calcium ( $3 \mathrm{mM})$, addition of gentamicin to the $\mathrm{PIP}_{2}$-enriched liposomes increased fluorescence significantly even when the concentration of the antibiotic was two orders of magnitude lower than that of calcium (Fig. 1C). Liposomes containing the other anionic phospholipids did not respond to the antibiotic at these calcium levels. Experiments without calcium yielded results identical to those obtained in the presence of $1 \mu \mathrm{M}$ calcium. In all cases, addition of calcium and aminoglycosides did not significantly alter the emission spectrum or shift the maximum emission wavelength of ANS.

When the ratio of $\mathrm{PC}$ to the anionic phospholipid was increased to $10: 1$ (Fig. 1D), liposomes containing $\mathrm{PIP}_{2}$ still reacted with gentamicin. Small increases in fluorescence were even noted for PC/PIP 2 liposomes with molar ratios as high as $15: 1$. In contrast, no increases in fluorescence were seen for other acidic phospholipids at

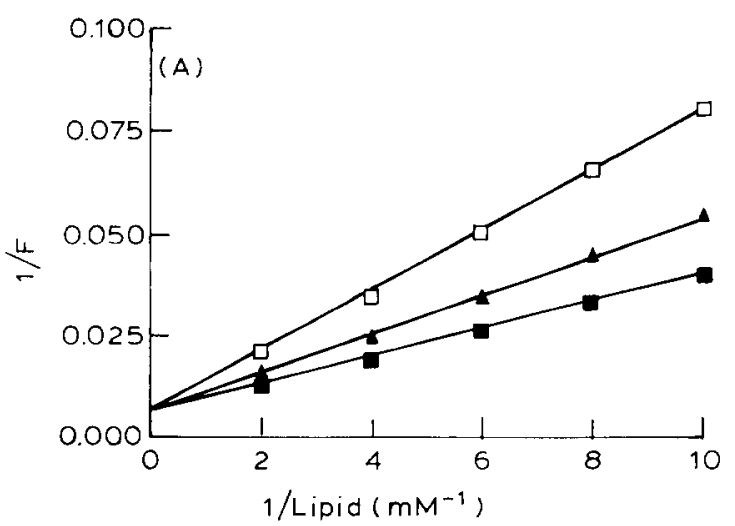

molar ratios of $8: 1$ (data not shown).

Analysis of the dependence of fluorescence on the liposome concentration showed that the extrapolated maximal fluorescence of $\mathrm{PC} / \mathrm{PIP}_{2}$ liposomes was unaffected by the aminoglycoside, gentamicin. This was also the case for amikacin, another aminoglycoside tested (Fig. 2A). With PC/PI, aminoglycosides induced significantly smaller changes of fluorescence but maximal fluorescence again remained unchanged.

Further quantitative analyses were performed for the binding parameters by a procedure similar to that described by Klotz [7]. If no interaction between the binding sites on the liposomes is assumed, the Klotz equation will take the form:

$$
\frac{L_{\mathrm{o}}}{x A_{\mathrm{o}}}=\frac{1}{n}+\left[\frac{K_{\mathrm{d}}}{n}\right]\left[\frac{1}{\left(A_{\mathrm{o}}-x A_{\mathrm{o}}\right)}\right]
$$

where:

$L_{\mathrm{o}}=$ the total liposome concentration;

$A_{\mathrm{o}}=$ the total ANS concentration;

$x=$ the fraction of the ANS bound (calculated as the ratio of the fluorescence value and the reciprocal of the intercept of the double-reciprocal plots of fluorescence versus liposome concentration in the presence of a given concentration of ANS);

$n=$ the number of binding sites (moles/mole phospholipid); and

$K_{\mathrm{d}}=$ the dissociation constant $(\mu \mathrm{M})$.

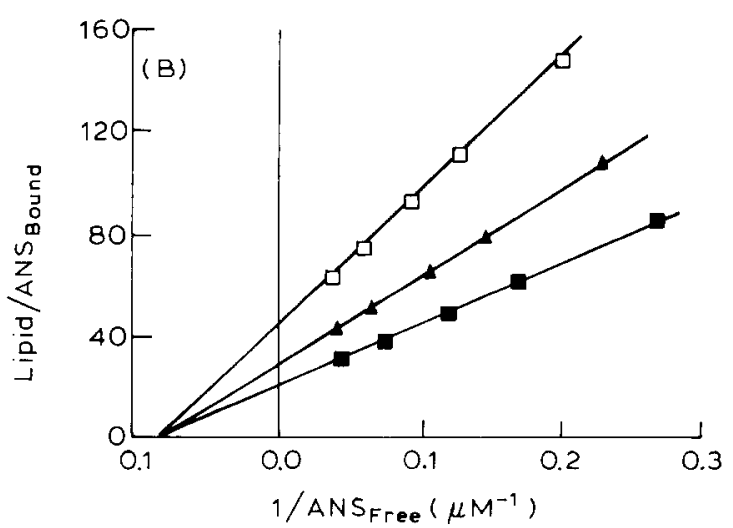

Fig. 2. (A) Double reciprocal plots of relative fluorescence against liposome concentration for PC/PIP 2 (molar ratio, $4: 1)$ liposomes

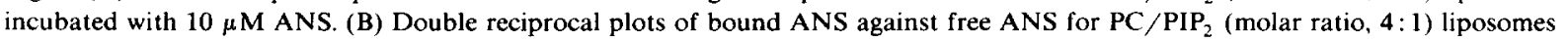

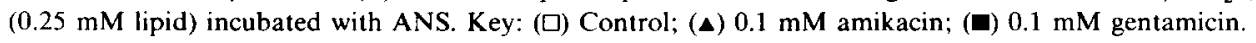


TABLE I

EFFECT OF AMINOGLYCOSIDES ON THE BINDING OF ANS TO LIPOSOMES OF PC AND PC/ANIONIC PHOSPHOLIPID (MOLAR RATIO, $4: 1$ )

\begin{tabular}{llll}
\hline $\begin{array}{l}\text { Liposome } \\
\text { composition }\end{array}$ & Aminoglycoside & $\begin{array}{l}n(\mathrm{~mol} \text { ANS } / \\
\text { mol PC) }\end{array}$ & $K_{\mathrm{d}}(\mu \mathrm{M})$ \\
\hline PC & None & 0.070 & 11.9 \\
PC $/$ PIP $_{2}$ & None & 0.029 & 11.8 \\
& Amikacin, 0.1 mM & 0.043 & 11.8 \\
& Gentamicin, $0.1 \mathrm{mM}$ & 0.063 & 12.1 \\
& Gentamicin, 0.3 mM & 0.068 & 11.8 \\
& Gentamicin, 0.5 mM & 0.070 & 12.1 \\
& Gentamicin, 0.7 mM & 0.071 & 11.9 \\
PC $/$ PI & None & 0.041 & 11.4 \\
& Gentamicin, 0.1 mM & 0.046 & 11.9 \\
\hline
\end{tabular}

Typical analyses are shown in Fig. $2 \mathrm{~B}$ and the binding parameters ( $n$ and $K_{\mathrm{d}}$ ) for $\mathrm{PC}, \mathrm{PC} / \mathrm{PIP}_{2}$ and $\mathrm{PC} / \mathrm{PI}$ liposomes in the absence and presence of aminoglycosides are summarized in Table I. In mixed liposomes, the number of ANS binding sites is lower than in pure PC liposomes. The addition of aminoglycoside, which does not affect PC liposomes, increases the number of ANS binding sites $(n)$ in these mixed liposomes but does not change the affinity of ANS to the lipid $\left(K_{\mathrm{d}}\right)$.

\section{Discussion}

The effects of calcium and aminoglycosides on membrane structure as determined by the membrane probe, ANS, are in excellent agreement with the conclusions from our studies of surface pressure of monolayers and differential scanning calorimetry of liposomes [2,3]. Phosphatidylinositol bisphosphate again was unique among the anionic lipids tested in its reactivity towards aminoglycosides. Even in the presence of calcium concentrations well above physiological significance and at ratios of calcium/drug $>100$, liposomes containing this lipid reacted with aminoglycosides. It also seems significant, in view of potential oto- and nephrotoxic mechanisms, that a drug interaction is observed at $\mathrm{PC} / \mathrm{PIP}_{2}$ ratios as low as 15:1 when other lipids were no longer reactive.

Beyond the information obtained previously, this new model gives insight into the aminoglyco- side-anionic phospholipid interaction at the molecular level. The increase in fluorescence by aminoglycosides could be due to changes in the molecular environment of the probe, in the affinity of the binding sites for ANS or in the number of binding sites available. The former effect would lead to a change in quantum yield of ANS while the latter two would lead to changes in the binding parameters.

The extrapolated maximum fluorescence of ANS in PC/PI and PC/PIP liposomes was the same and was unaffected by the addition of aminoglycosides (Fig. 2A). Thus, the drugs did not change the quantum yield $(Q)$. The facts that neither the aminoglycosides or the nature of the acidic lipid caused a quantum yield change or a change in the ANS emission spectrum suggest that these alterations do not change the microenvironment of an individual ANS molecule bound to PC. This conclusion is further supported by the observation that neither the addition of an anionic lipid or an aminoglycoside affected the affinity of ANS for its binding site $\left(K_{\mathrm{d}}\right)$ (Table I). The constancy of both the $K_{\mathrm{d}}$ and $Q$ values in the presence and absence of an anionic lipid is in excellent agreement with the assumption that ANS binds mainly to $\mathbf{L}-\boldsymbol{\alpha}$-phosphatidylcholine [8] both in the presence and absence of aminoglycoside.

The increases in fluorescence of the PC/PI and $\mathrm{PC} / \mathrm{PIP}_{2}$ liposomes upon treatment with different aminoglycosides were due entirely to increases in the number of ANS binding sites (Table I). ANS binds to phospholipids in the region of their polar head groups and the number of molecules bound is determined by the surface charge of the liposome [9-11] and inversely proportional to the magnitude of the negative membrane surface potential [12]. Charged phospholipids contribute little to ANS binding [13,14], but the presence of cations increases ANS fluorescence; trivalent cations are more effective than bivalent cations which in turn are more active than univalent ones $[15,16]$. Thus, reductions in the electrostatic surface potential facilitate the binding of ANS molecules to the liposome $[16,17]$. The differences in the number of binding sites in the PC, PC/PI and $\mathrm{PC} / \mathrm{PIP}_{2}$ liposomes observed here (Table I) should reflect differences in the surface potentials introduced by the anionic lipids resulting in a masking 
of the binding sites for ANS on PC. This agrees with the conclusion reached above that ANS binds to the same receptor site, i.e., the same lipid, in all the liposomal systems tested.

Gentamicin increased by $117 \%$ the number of binding sites in $\mathrm{PC} / \mathrm{PIP}_{2}$ liposomes but only by $12 \%$ in PC/PI liposomes (Table I). Gentamicin was much more effective than an equivalent concentration of amikacin. These results are consistent with our previous findings that related the degree of interaction of aminoglycosides with $\mathrm{PIP}_{2}$ to their ototoxicity [2]. The effects of aminoglycosides can not be explained on the basis of charge neutralization of the liposomes only. For example, netilmicin is far more effective than amikacin in neutralizing surface charge as measured by the zeta potential of $\mathrm{PC} / \mathrm{PIP}_{2}$ liposomes, but is less effective in increasing ANS fluorescence [6]. Furthermore, aminoglycoside effects with $\mathrm{PIP}_{2}$ are seen at high calcium/low aminoglycoside concentrations (ratio, 100:1) where the drug contribution to charge neutralization should be negligible. Such an effect is not obtained when PI is used in

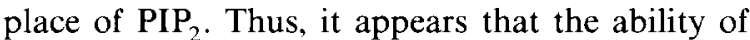
the aminoglycosides to increase ANS fluorescence is dependent on a specific $\mathrm{PIP}_{2}$-aminoglycoside interaction. This may be the induction of phase separation and the creation of large domains rich in PC. By such a mechanism, the number of potential binding sites for ANS will increase as an environment of pure $\mathrm{PC}$ is created which is not masked by anionic phospholipid. This would explain the fact that only the value of $n$ (number of unscreened sites), but not the values of $K_{\mathrm{d}}$ (affinity of the sites) and $Q$ (reflecting the microenvironment of the probe) are affected by the aminoglycosides. Finally, the postulate of aminoglycoside-induced phase separation is strengthened by the observation that exposure of the $\mathrm{PC} / \mathrm{PIP}_{2}$ liposomes to higher concentrations of gentamicin $(0.3-0.7 \mathrm{mM})$ results in a maximal $n$ of 0.07 , a value equal to the number of binding sites in pure PC liposomes (Table I). This interpretation is con- sistent with data obtained by differential scanning calorimetry which suggested the ability of aminoglycosides to induce phase separation in PC/ PIP 2 liposomes [3].

These findings further support our hypothesis that the polar head group of phosphatidylinositol bisphosphate is a specific site of action for aminoglycosides. The perturbation of $\mathrm{PIP}_{2}$-containing membranes by aminoglycosides and the resulting changes in membrane structure and function therefore may play a crucial role in the ototoxicity and nephrotoxicity of these drugs.

\section{Acknowledgement}

This study was supported by grant NS-13792 from the National Institutes of Health.

\section{References}

1 Schacht, J. and Weiner, N. (1986) ORL 48, 116-123

2 Wang, B.M., Weiner, N.D., Takada, A. and Schacht, J. (1984) Biochem. Pharmacol. 33, 3257-3252

3 Wang, B.M., Weiner, N.D., Ganesan, M.G. and Schacht, J. (1984) Biochem. Pharmacol. 33, 3787-3791

4 Slavik, J. (1982) Biochim. Biophys. Acta 694, 1-25

5 Schacht, J. (1978) J. Lipid Res. 19, 1063-1067

$6 \mathrm{Au}, \mathrm{S}$., Weiner, N. and Schacht, J. (1986) Antimicrob. Chemother. 30, 395-397

7 Klotz, J. (1947) Chem. Rev. 41, 373-399

8 Haynes, D.H. and Staerk, H. (1974) J. Membrane Biol. 17 , $313-340$

9 Radda, G.K. (1975) in Methods in Membrane Biology (Korn, E.D., ed.), Vol. 4, pp. 97-188, Plenum Press, New York

10 Kapoor, M. (1976) Biochem. Rev. 47, 27-51

11 Fortes, P.A.G. and Hoffman, J.F. (1971) J. Membrane Biol. $5,154-168$

12 Azzi, A. (1975) Q. Rev. Biophys. 8, 237-316

13 Feinstein, M.B., Spero, L. and Falsenfeld, H. (1970) FEBS Lett. 6, 245-248

14 Bashford, C.L., Morgan, C.G. and Radda, G.K. (1976) Biochim. Biophys. Acta 426, 157-172

15 Gomperts, B., Lantelme, F. and Stock, R. (1970) J. Membrane Biol. 3, 241-246

16 Haynes, D.H. (1974) J. Membrane Biol. 17, 341-366

17 Schäfer, G. and Rowohl-Quisthoudt, G. (1975) FEBS Lett $59,48-51$ 